\title{
STRONGLY EXPOSED POINTS IN WEAKLY COMPACT CONVEX SETS IN BANACH SPACES
}

\author{
J. BOURGAIN
}

\begin{abstract}
A "purely geometric" proof of the Lindenstrauss-Troyanski result ([2], [6]) on strongly exposed points of weakly compact sets in Banach spaces is given.

I would like to thank the referee for his suggestions.
\end{abstract}

We consider a Banach-space $X$, \|\| . If $\xi \in X$ and if $\varepsilon>0$, then $B(\xi, \varepsilon)$ $=\{x \in X ;\|x-\xi\|<\varepsilon\}$. The closed convex hull of a set $A \subset X$, is denoted $\bar{c}(A)$. Let $C$ be a convex subset of $X$, then $C^{e}$ is the set of the extremal points of $C$. If $K$ is a convex, weakly compact, $B$ a convex, closed, bounded set of $X$ and $J$ a closed subinterval of $[0,1],(K, B, J)$ denotes the closed, convex set $\{(1-t) k+t b ; k \in K, b \in B, t \in J\}$.

We have to introduce a few geometrical definitions, referring to [4].

Suppose that $C$ is a nonempty, bounded, closed and convex subset of $X$. Let $M(C)=\sup \{\|x\| ; x \in C\}$. If $f \in X^{*}$ with $\|f\|=1$, let $M(f, C)$ $=\sup \{f(x) ; x \in C\}$, and for each $\alpha>0$, let $S(f, \alpha, C)=\{x \in C ; f(x)$ $\geqslant M(f, C)-\alpha\}$. Such a set is called a "slice" of $C$. A point $\xi$ of $C$ is called "strongly exposed" if there exists $f \in X^{*}(\|f\|=1)$ such that $\forall \varepsilon>0, \exists \alpha$ $>0$ with $\xi \in S(f, \alpha, C) \subset B(\xi, \varepsilon)$. Let $S$ be the set of all $f \in X^{*}$ such that $\|f\|=1$ and $f$ strongly exposes some point of $C$.

Proposition 1. If $K$ is a nonempty, convex, weakly-compact subset of $X$ and if $B$ is convex, closed and bounded with $K \cap B=\varnothing$, then the set $D=\bar{c}(K \cup B)$ $=(K, B,[0,1])$ has the following property: $\forall \varepsilon>0, \exists \xi \in K$ such that $\xi$ $\notin \bar{c}(D \backslash B(\xi, \varepsilon))$.

First we observe that it is sufficient to prove the proposition for $X$ separable. Indeed, suppose $D$ does not have the required property. Then there exists $\varepsilon>0$ with $\forall x \in K: x \in \bar{c}(D \backslash B(x, \varepsilon))$ and hence, $\forall x \in K, \exists A(x) \subset D$ with the properties: $x \in \bar{c}(A(x)), A(x) \cap B(x, \varepsilon)=\varnothing$ and $A(x)$ is countable. By induction we construct a sequence $\left(K_{n}, B_{n}\right)_{n}$ where $K_{n}$ is countable in $K$ and $B_{n}$ is countable in $B$.

Let $\left(K_{0}, B_{0}\right)=(\{x\}, \varnothing)$, where $x$ is some element of $K$. Suppose we already found $\left(K_{n}, B_{n}\right)$.

Consider $\forall y \in \cup_{x \in K_{n}} A(x)$ an element $k_{y}$ in $K$ and $b_{y}$ in $B$, with $y$ $\in \bar{c}\left(\left\{k_{y}, b_{y}\right\}\right)$.

Let

Received by the editors September 29, 1975.

AMS (MOS) subject classifications (1970). Primary 46B10; Secondary 46B99. 


$$
\begin{array}{r}
K_{n+1}=\left\{\sum_{i=1}^{m} \lambda_{i} x_{i}, m \in \mathbf{N}^{*}, x_{i} \in K_{n} \cup \bigcup_{x \in K_{n}}\left\{k_{y} ; y \in A(x)\right\},\right. \\
\left.\lambda_{i} \in \mathbf{Q}^{+}(1 \leqslant i \leqslant m) \text { and } \sum_{i=1}^{m} \lambda_{i}=1\right\},
\end{array}
$$

and

$$
\begin{array}{r}
B_{n+1}=\left\{\sum_{i=1}^{m} \lambda_{i} x_{i} ; m \in \mathbf{N}^{*}, x_{i} \in B_{n} \cup \bigcup_{x \in K_{n}}\left\{b_{y} ; y \in A(x)\right\},\right. \\
\left.\lambda_{i} \in \mathbf{Q}^{+}(1 \leqslant i \leqslant m) \text { and } \sum_{i=1}^{m} \lambda_{i}=1\right\} .
\end{array}
$$

Then $K_{n+1}$ and $B_{n+1}$ are still countable, which completes the construction.

Consider then $K^{\prime}=\overline{\cup_{n} K_{n}}, B^{\prime}=\overline{\cup_{n} B_{n}}, D^{\prime}=\bar{c}\left(K^{\prime} \cup B^{\prime}\right)$ and the closed linear span $X_{0}$ of $D^{\prime}$.

Since $\forall n: \bar{c}\left(K_{n}\right) \subset \bar{K}_{n+1}, K^{\prime}$ is convex and closed. The same holds for $B^{\prime}$. $X_{0}$ is a separable Banach-space wherein $K^{\prime}$ is nonempty, convex, weaklycompact and $B^{\prime}$ convex, closed and bounded. We show that $D^{\prime} \subset X_{0}$ does not have the property mentioned in the proposition. Choose $x^{\prime} \in K^{\prime}$ and take $0<\delta<\varepsilon / 2$. There exist $n \in \mathbf{N}$ and $x \in K_{n}$ with $\left\|x-x^{\prime}\right\| \leqslant \delta$. We obtain:

$$
\begin{aligned}
x \in \bar{c}(A(x)) & \subset \bar{c}\left(\bigcup_{y \in A(x)} \bar{c}\left(\left\{k_{y}, b_{y}\right\}\right) \backslash B(x, \varepsilon)\right) \subset \bar{c}\left(D^{\prime} \backslash B(x, \varepsilon)\right) \\
& \subset \bar{c}\left(D^{\prime} \backslash B\left(x^{\prime}, \varepsilon / 2\right)\right) .
\end{aligned}
$$

Since this holds, $\forall \delta(0<\delta<\varepsilon / 2)$, we have $x^{\prime} \in \bar{c}\left(D^{\prime} \backslash B\left(x^{\prime}, \varepsilon / 2\right)\right)$. $D^{\prime}$ does not have the property and we can restrict ourselves to the case of a separable Banach-space.

LEMMA. $D=\bar{c}\left(D^{e} \cup B\right)$.

Proof. Clearly $E=\bar{c}\left(D^{e} \cup B\right) \subset D$. For the reverse inclusion it is sufficient to prove that $K \subset E$. Suppose that $x_{0} \in K \backslash E$. Then $\exists u \in X^{*}$ such that $u\left(x_{0}\right)>\sup \left\{u(x) ; x \in D^{e} \cup B\right\} \geqslant \sup \{u(x) ; x \in B\}$. Let $\alpha=\sup \{u(x) ; x$ $\in K\}$, and let $y \in D$. Then $y=\lambda x_{1}+(1-\lambda) x_{2}$ with $x_{1} \in K, x_{2} \in B, \lambda$ $\in[0,1]$. It follows that

$$
\begin{aligned}
u(y) & =\lambda u\left(x_{1}\right)+(1-\lambda) u\left(x_{2}\right) \leqslant \lambda u\left(x_{1}\right)+(1-\lambda) u\left(x_{0}\right) \\
& =u\left(\lambda x_{1}+(1-\lambda) x_{0}\right) \leqslant \alpha .
\end{aligned}
$$

This shows that $\sup \{u(y) ; y \in D\}=\alpha$, and, since $u\left(x_{2}\right)<u\left(x_{0}\right) \leqslant \alpha, u(y)$ $=\alpha$ implies that $\lambda=1$ or $y \in K$. Let $D_{1}=\{y \in D ; u(y)=\alpha\}$. Then $D_{1}$ is a supporting set of $D$, and $D_{1} \subset K$. Since $D_{1}$ is weakly compact, it contains an extreme point $z$. Then $z \in D^{e}$, but since $u(z)=\alpha, z \notin\left(D^{e} \cup B\right)$. This contradiction establishes that $K \subset E$ or $D=E$.

Proof of Proposition 1. The proof is a modification of a proof in Namioka [3]. We assume that $X$ is separable. By the lemma, $A=D^{e} \backslash B \neq \varnothing$. Since $A \subset K$ the weak closure $\bar{A}$ of $A$ is a Baire space relative to the weak topology. Since $X$ is separable, there is a sequence $\left\{x_{n}\right\}$ in $X$ such that 


$$
\bar{A} \subset \bigcup_{n=1}^{\infty} \bar{B}\left(x_{n}, \varepsilon / 4\right) \text {. }
$$

Note that each $\bar{B}\left(x_{n}, \varepsilon / 4\right)$ is weakly closed. Hence there is a weakly open set $N$ in $X$ such that $N \cap \bar{A} \neq \varnothing$ and $N \cap \bar{A} \subset \bar{B}\left(x_{n}, \varepsilon / 4\right)$ for some $n$. Then clearly $\operatorname{diam}(N \cap \bar{A}) \leqslant \varepsilon / 2$. Let

$$
D_{1}=\bar{c}(N \cap \bar{A}) \quad \text { and } \quad D_{2}=\bar{c}((\bar{A} \backslash N) \cup B) .
$$

Then we observe that $D_{1}$ is weakly compact and diam $D_{1} \leqslant \varepsilon / 2$.

Since $A$ is weakly dense in $A, N \cap A \neq \varnothing$. Fix $\xi \in N \cap A \subset K$. Then $\xi \notin D_{2}$. For if $\xi \in D_{2}$, then $\xi \in \bar{c}((K \backslash N) \cup B) \subset(\bar{c}(K \backslash N), B,[0,1])$. Since $\xi \in D^{e}$ and $\xi \notin B$, we have $\xi \in \bar{c}(K \backslash N)$. It then follows from the Krein-Milman theorem that $\xi \in K \backslash N$, because $K \backslash N$ is weakly compact. This contradicts $\xi \in N \cap A$.

Because $\quad D=\bar{c}\left(D^{e} \cup B\right), D=\bar{c}\left(D_{1} \cup D_{2}\right)=\left(D_{1}, D_{2},[0,1]\right)$. Let $C$ $=\left(D_{1}, D_{2},[\varepsilon / 5 d, 1]\right)$, where $d=\operatorname{diam} D$. Then $C$ is a closed convex subset of $D$.

If $\xi \in C$, then $\xi=(1-\lambda) x_{1}+\lambda x_{2}$ where $x_{1} \in D_{1}, x_{2} \in D_{2}$ and $\lambda$ $\in[\varepsilon / 5 d, 1]$. Since $\xi$ is extreme, this implies that $\xi \in D_{2}$, which is impossible. Hence $\xi \notin C$. Let $y_{1}, y_{2} \in D \backslash C$. Then $y_{i}=\left(1-\lambda_{i}\right) x_{1}^{i}+\lambda_{i} x_{2}^{i}$, where $x_{1}^{i}$ $\in D_{1}, x_{2}^{i} \in D_{2}$ and $\lambda_{i} \in[0, \varepsilon / 5 d](i=1,2)$. We then have:

$$
\begin{aligned}
\left\|y_{1}-y_{2}\right\| & \leqslant\left\|x_{1}^{1}-x_{1}^{2}\right\|+\lambda_{1}\left\|x_{1}^{1}-x_{2}^{1}\right\|+\lambda_{2}\left\|x_{1}^{2}-x_{2}^{2}\right\| \\
& \leqslant \varepsilon / 2+\varepsilon d / 5 d+\varepsilon d / 5 d=9 \varepsilon / 10 .
\end{aligned}
$$

Since $\xi \in D \backslash C$ and diam $(D \backslash C)<\varepsilon$, it follows that $D \backslash C \subset B(\xi, \varepsilon)$. Therefore $D \backslash B(\xi, \varepsilon) \subset C$ and $\bar{c}(D \backslash B(\xi, \varepsilon)) \subset C$. Thus $\xi \notin \bar{c}(D \backslash B(\xi, \varepsilon))$ and the proof is complete.

Remark. The fact that $X,\|\|$ is complete is not used and the assertion is still true when $X$ is only a normed space.

Proposition 2. Let $C$ be a convex and weakly-compact subset of $X$. If $S(f, \alpha, C)$ is a slice of $C$, then $\forall \varepsilon>0: \exists g \in X^{*}, \exists \beta>0$ such that $S(g, \beta, C)$ is a slice of $C$ with diameter $\leqslant \varepsilon, S(g, \beta, C) \subset S(f, \alpha, C)$ and $\|f-g\| \leqslant \varepsilon$.

Proof. The proof is a modification of a proof in Phelps [4]. By translation we may assume that $0 \in H=\{x \in X ; f(x)=M(f, C)-\alpha\}$. Hence $H$ $=f^{-1}(0)$ and $\alpha=M(f, C)>0$. We may also assume that $\varepsilon<\min (1, \alpha)$. Choose $\lambda$ so that $\lambda>2 M(C) / \varepsilon$, and let $K=S(f, \alpha / 2, C)$ and $B=H$ $\cap \bar{B}(0, \lambda)$. Since $K \cap B=\varnothing$ and $K \neq \varnothing$, we may apply Proposition 1 to $K$ and $B$.

Let $D=\bar{c}(K \cup B)$. Then $\exists \xi \in K$ such that $\xi \notin \bar{c}(D \backslash B(\xi, \varepsilon / 2))$. If $x \in B$, then $\|x-\xi\| \geqslant|f(x-\xi)| \geqslant \alpha / 2>\varepsilon / 2$. Therefore $B \subset \bar{c}(D \backslash B(\xi, \varepsilon / 2))$. By the separation theorem, $\exists g \in X^{*}$ such that $\|g\|=1$ and $g(\xi)>\sup \{g(x) ; x$ $\in D \backslash B(\xi, \varepsilon / 2)\} \geqslant 0$. Since $M(g, C) \geqslant g(\xi)$, we may write $\sup \{g(x) ; x$ $\in D \backslash B(\xi, \varepsilon / 2)\}=M(g, C)-2 \beta$ with $\beta>0$.

Then $S(g, \beta, C) \subset B(\xi, \varepsilon / 2)$, and hence diam $S(g, \beta, C) \leqslant \varepsilon$. Suppose that $f(x)=0$ and $\|x\| \leqslant 1$. Then $\lambda x \in B$, and hence $\lambda g(x)<g(\xi)$ or $g(x)$ $<\lambda^{-1} g(\xi)$. By Lemma 2 of [1], this implies that either $\|f-g\| \leqslant 2 \lambda^{-1} g(\xi)$ 
$\leqslant 2 \lambda^{-1} M(C)<\varepsilon$ or $\|f+g\| \leqslant 2 \lambda^{-1} g(\xi)$. If the second possibility occurs, then $g(\xi)<(f+g)(\xi) \leqslant\|f+g\|\|\xi\| \leqslant 2 \lambda^{-1} g(\xi) M(C)<g(\xi) \varepsilon$, which implies that $1<\varepsilon$. But we assumed $\varepsilon<1$. Therefore $\|f-g\|<\varepsilon$.

THEOREM. Let $C$ be a convex and weakly-compact subset of $X$. Then $C$ is the closed convex hull of its strongly exposed points and the set $S$, defined in the introduction, is a dense $G_{\delta}$ subset of the unit sphere $\left\{f \in X^{*} ;\|f\|=1\right\}$ of $X^{*}$.

Proof. Referring to Lemma 7 of [4], the first assertion is a consequence of Proposition 2. We remark that it also follows from the second part of the theorem.

For $\varepsilon>0$, let $U(\varepsilon)$ be the set of all $f \in X^{*}$ such that $\|f\|=1$ and $\operatorname{diam} S(f, \alpha, C) \leqslant \varepsilon$ for some $\alpha>0$. Then $U(\varepsilon)$ is an open subset of the unit sphere of $X^{*}$. Indeed, suppose $f \in U(\varepsilon)$ and $\operatorname{diam} S(f, \alpha, C) \leqslant \varepsilon$, then we verify that $S(g, \alpha / 3, C) \subset S(f, \alpha, C)$ if $\|g\|=1$ and $\|f-g\|<\alpha / 3 M(C)$. It is clear from Proposition 2 that $U(\varepsilon)$ is also dense there.

Since $S=\cap_{n=1}^{\infty} U(1 / n), S$ is a dense $G_{\delta}$ subset of the unit sphere by the Baire category theorem.

\section{BIBLIOGRAPHY}

1. E. Bishop and R. R. Phelps, The support functionals of a convex set, Proc. Sympos. Pure Math., vol. 7, Amer. Math. Soc., Providence, R.I., 1963, pp. 27-35. MR 27 \# 4051.

2. J. Lindenstrauss, On operators which attain their norm, Israel J. Math. 1 (1963), 139-148. MR 28 \#3308.

3. I. Namioka, Neighbourhoods of extreme points, Israel J. Math. 5 (1967), 145-152. MR 36 \#4323.

4. R. R. Phelps, Dentability and extreme points in Banach spaces, J. Functional Analysis 17 (1974), 78-90. MR 50 \#5427.

5. Lectures on Choquet's theorem, Van Nostrand, Princeton, N.J., 1966. MR 33 $\# 1690$.

6. S. L. Trojanski, On locally uniformly convex and differentiable norms in certain non-separable Banach spaces, Studia Math. 37 (1970/71), 173-180. MR 46 \# 5995.

Department of Mathematics, Vrije Universiteit Brussels, Brussels, Belgium 